\title{
Sustained Visual Attention Performance-Associated Prefrontal Neuronal Activity: Evidence for Cholinergic Modulation
}

\author{
T. Michael Gill, Martin Sarter, and Ben Givens \\ Department of Psychology, The Ohio State University, Columbus, Ohio 43210
}

Cortical cholinergic inputs are hypothesized to mediate attentional functions. The present experiment was designed to determine the single unit activity of neurons within the medial prefrontal cortex (mPFC) of rats performing a sustained visual attention task. Demands on attentional performance were varied by the presentation of a visual distractor. The contribution of cholinergic afferents of the mPFC to performance-associated unit activity within this area was determined by recording neuronal activity before and after unilateral cholinergic deafferentation using intracortical infusion of the immunotoxin 192 lgGsaporin. Presentation of the visual distractor resulted in a decrease in the detection of brief, unpredictable visual signals. As predicted, the unilateral loss of cholinergic inputs within the recording area of the $\mathrm{mPFC}$ did not affect sustained attentional performance. Cholinergic deafferentation, however, resulted in a decrease in the overall firing rate of medial prefrontal neurons and a substantial reduction in the proportion of neurons whose firing patterns correlated with specific aspects of behavioral performance. Furthermore, cholinergic deafferentation attenuated the frequency and amplitude of increased mPFC neuronal firing rates that were associated with the presentation of the visual distractor. The main findings from this experiment suggest that cholinergic inputs to the mPFC strongly influence spontaneous and behaviorally correlated single unit activity and mediate increases in neuronal activity associated with enhanced demands for attentional processing, all of which may be fundamental aspects in the maintenance of attentional performance.

Key words: medial prefrontal cortex; attention; acetylcholine; basal forebrain; 192 lgG-saporin; cholinergic
Cortical cholinergic afferents arising from the basal forebrain (BF) have been hypothesized to modulate the processing of stimuli and associations that may ultimately lead to effective cognitive performance (Everitt and Robbins, 1997; Robbins, 1997; Sarter and Bruno, 1997, 1999). Lesion-induced loss of BF neurons interferes with performance in tasks designed to assess various aspects of attentional functions (Robbins et al., 1989; Muir et al., 1992, 1993, 1994; Voytko, 1996). Recent experiments have demonstrated that the selective loss of corticopetal cholinergic projections is sufficient to produce impairments in attention (Chiba et al., 1995, 1999; Bucci et al., 1998), especially in sustained (McGaughy et al., 1996; McGaughy and Sarter, 1998) and divided attention (Turchi and Sarter, 1997). In intact animals, inf usions of drugs into the BF known to bidirectionally modulate the excitability of cholinergic corticopetal projections produce correlated changes in attentional performance (Sarter et al., 1999), further supporting the role of this neuronal system in the mediation of fundamental aspects of information processing and suggesting that aberrations in the regulation of this cortical projection system profoundly affect cognitive functions (Sarter and Bruno, 1999).

Studies assessing cortical acetylcholine (ACh) release in attentional task-performing rats have generated preliminary evidence

\footnotetext{
Received Jan. 10, 2000; revised April 6, 2000; accepted April 6, 2000.

This work was supported by National Institutes of Health (NIH) Grant NS37026 to M.S. and B.G. and NIH training Grant MH19936 to T.M.G. We thank Dave Kent, Suzanna Yan, and Helen Sabolek for their valuable assistance with behavioral training and data collection.

Correspondence should be addressed to Dr. B. Givens, Department of Psychology, 1885 Neil Avenue, Ohio State University, Columbus, OH 43210. E-mail: givens+@osu.edu.

Copyright (c) 2000 Society for Neuroscience $\quad 0270-6474 / 00 / 124745-13 \$ 15.00 / 0$
}

suggesting that demands on sustained attention performance are associated with increases in cortical ACh efflux (Himmelheber et al., 1998) and specifically within the prefrontal cortex (Sarter et al., 1996), a brain area strongly implicated in the executive control of cognitive processes (Robbins, 1996). Although prefrontal neural activity has been extensively associated with the control of attentional mechanisms and other aspects of cognitive processing in monkeys (Fuster, 1990; Watanabe, 1990; Goldman-Rakic, 1994), and even though there have been a few rodent studies that have examined prefrontal neuronal activity during spatial and nonspatial learning tasks (Schoenbaum and Eichenbaum, 1995; Chang et al., 1997; Poucet, 1997; Jung et al., 1998), none have specifically examined prefrontal activity during attentional processing or the dependence of that activity on corticopetal cholinergic input in rats. Thus, the present experiment was designed to assess neuronal activity within the medial prefrontal cortex (mPFC), using in vivo neurophysiological recording techniques, in rats performing a sustained visual attention task and to determine the contribution of cholinergic input to performance-associated neuronal activity before and after cholinergic deafferentation of mPFC using local inf usions of 192 IgG-saporin [for a validation of this method see Holley et al. (1994) and Bucci et al. (1998)]. Importantly, previous pilot studies have indicated that unilateral cortical cholinergic deafferentation of the mPFC, as opposed to more extensive loss of cortical cholinergic inputs [for discussion, see Sarter and Bruno (1997)], does not suffice to affect performance in this task, thereby allowing the determination of the contribution of cholinergic inputs to $\mathrm{mPFC}$ neural activity without the potentially confounding effects of impairments in behavioral performance. Medial prefrontal neuronal activity was expected to exhibit distinct relationships with individual aspects of behavioral performance and demands on attentional processing, 
and the latter was specifically hypothesized to depend on intact corticopetal cholinergic input.

\section{MATERIALS AND METHODS}

\section{Subjects}

The subjects were adult male Long-Evans rats $(n=9)$ (Harlan Sprague Dawley, Indianapolis, IN) that weighed 200-250 gm at the start of the experiment. The rats were housed in pairs before surgery and then individually housed after surgery within a climate-controlled vivarium $\left(25^{\circ} \mathrm{C}\right)$ on a $12 \mathrm{hr}$ light/dark cycle (lights on at 7 A.M.). Food and water were available ad libitum until commencement of behavioral testing, after which the rats were allowed limited access to water in their home cage $(10-20 \mathrm{~min} / \mathrm{d})$ after behavioral testing to maintain at least $85 \%$ of their unrestricted body weight. The research was conducted in a facility approved and accredited by the American Association of Accreditation of Laboratory Animal Care and in accordance with U.S. Government Principles for the Utilization and Care of Vertebrate Animals Used in Testing, Research and Training (Public Health Service, 1996).

\section{Behavioral apparatus}

Sustained visual attention training and testing occurred in two different types of operant chambers (Med Associates, East Fairfield, VT). Initial behavioral training was performed in standard operant chambers $(28 \mathrm{~cm}$ length $\times 21 \mathrm{~cm}$ width $\times 27 \mathrm{~cm}$ height) located inside light and sound attenuating shells $(64 \mathrm{~cm} \times 41 \mathrm{~cm} \times 41 \mathrm{~cm})$. The front panel of each chamber contained three signal lights $(2.8 \mathrm{~W})$ centered $6 \mathrm{~cm}$ above three response levers. Each response lever was located $7 \mathrm{~cm}$ above a grid floor. A tone generator was located on the back panel along with a water dispenser that delivered a single drop of water $(40 \mu \mathrm{l})$ into a recessed water port $(5 \mathrm{~cm}$ width $\times 3 \mathrm{~cm}$ depth $\times 5 \mathrm{~cm}$ height $)$. The operant chambers were illuminated by a houselight $(2.8 \mathrm{~W})$ located $5 \mathrm{~cm}$ above the central signal light on the front panel. A separate operant chamber was custom-built for neurophysiological recording and was identical to the training chambers except that it had taller side walls $(42 \mathrm{~cm})$, a larger recessed water port to accommodate the head-mounted recording equipment $(6.5 \mathrm{~cm} \times 6 \mathrm{~cm} \times 13 \mathrm{~cm})$, an infrared diode emitter and detector at the entrance to the water port, and was housed in a larger light and sound attenuating shell $(59 \mathrm{~cm} \times 39 \mathrm{~cm} \times 54 \mathrm{~cm})$. A CCD video camera was mounted through the top of the shell and was connected to a monitor and video cassette recorder with which behavior was observed and recorded. Both types of operant chambers were controlled by personal computers interfaced with hardware and software developed by Med Associates.

\section{Behavioral training}

All rats started behavioral testing after 1-2 weeks of acclimation to the vivarium and daily handling. The rats were initially trained on a twolever press paradigm using an FR-1 schedule of water reinforcement until they emitted 50 responses on both the left and right response levers (center lever responses were inconsequential). After initial shaping, behavioral training was conducted in four stages, each of which required the rats to discriminate the presence of brief, unpredictable signal events (illumination of the central signal light) from nonsignal events (nonillumination of central signal light). Both signal and nonsignal events were followed $1 \mathrm{sec}$ later by a tone $(200 \mathrm{msec})$ that initiated a $4 \mathrm{sec}$ response window during which bar presses were scored as correct or incorrect based on the following rules: (1) left lever responses on signal trials were correct, scored as "hits," and were followed by water reinforcement; (2) right lever responses on signal trials were incorrect, scored as "misses," and were not followed by reinforcement; (3) right lever responses on nonsignal trials were correct, scored as "correct rejections," and were followed by water reinforcement; (4) left lever responses on nonsignal trials were incorrect, scored as "false alarms," and were not followed by reinforcement. The front panel of the operant chambers and the response rules of the task are schematically represented in Figure 1 (see also McGaughy and Sarter, 1995). Bar presses within the response window or the expiration of the response window without an emitted response initiated a variable intertrial interval (ITI) $(10 \pm 3 \mathrm{sec})$. An equal number of signal and nonsignal trials were pseudorandomly presented within a testing session. Each behavioral testing session included a 36 min task period, divided into three 12 min blocks of trials, that was both preceded and followed by $5 \mathrm{~min}$, task-free periods. Training was conducted 5-6 d/week throughout all four stages, and each rat was required to reach criterion performance before advancing to the next

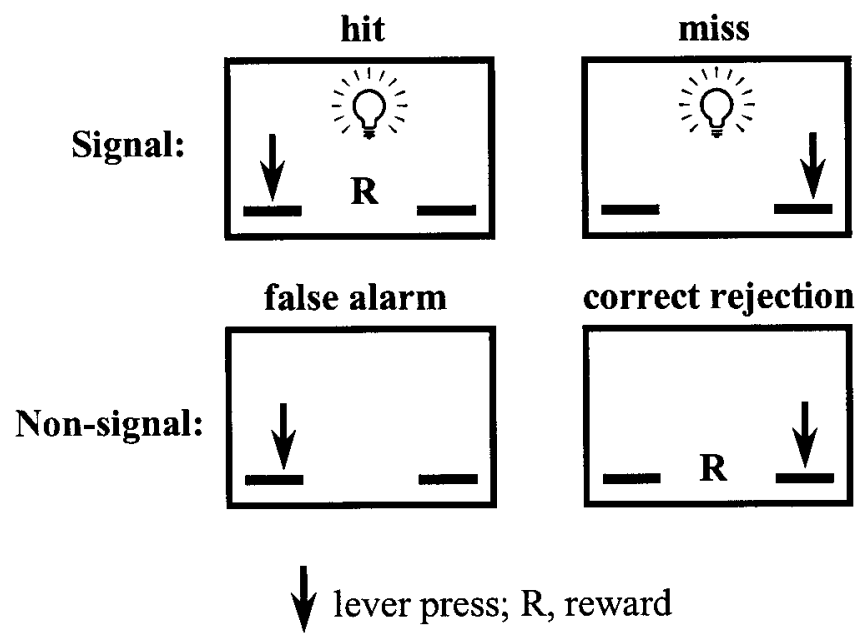

Figure 1. Response rules of the sustained visual attention task. Schematic of the response rules of the sustained visual attention task after the presentation of signal or nonsignal events. The top two panels demonstrate the response rules after center panel light illumination (signal event). The bottom two panels demonstrate the response rules after the nonillumination of the center panel light (nonsignal event). A tone was presented $1 \mathrm{sec}$ after signal and nonsignal events initiating a $4 \mathrm{sec}$ response window. Water reinforcement $(40 \mu \mathrm{l})$ was delivered into a water port affixed to the back wall of the operant chamber.

stage. The criterion performance level for the first three stages of behavioral training was a response accuracy of at least $70 \%$ on both signal and nonsignal trials, with $<30 \%$ total omissions for 3 consecutive days.

The first stage of behavioral training required the rats to discriminate between the presence and absence of $500 \mathrm{msec}$ signal light illuminations. To facilitate behavioral performance at this stage of training, correction trials were inserted after incorrect responses in which the same trial was presented again until a correct response was emitted, or until a maximum of five trial repetitions had occurred. The second stage of training was identical to the first stage except for the absence of correction trials after incorrect responding. The third stage of training required the discrimination between the presence and absence of signals that varied in length $(25,50$, and $500 \mathrm{msec})$. All three signal lengths were pseudorandomly selected and equally presented throughout each testing session. The last stage of training was conducted in the neurophysiological recording chamber and required the same type of signal discriminations as stage three; however, criterion performance at this stage was slightly more stringent and consisted of a response accuracy of at least $75 \%$ correct on both signal and nonsignal trials with $<25 \%$ omissions for 3 consecutive days. After reaching criterion performance, the rats continued training on standard testing sessions and on every fourth day were tested on a distractor version of the attentional task. The only difference between the two types of testing sessions was that during distractor testing sessions the houselight flashed at $0.5 \mathrm{~Hz}$ during the second 12 min block of trials. Each rat received approximately six distractor sessions before surgical implantation of recording electrodes into the mPFC.

\section{Recording electrodes}

Multiple unit activity within the mPFC was recorded using a pair of tungsten wires (20 $\mu \mathrm{m}$ diameter; California Fine Wire, Grover Beach, CA) that were immersed in epoxy and baked at $200^{\circ} \mathrm{C}$ for $1 \mathrm{hr}$. Two pair of fine wires were inserted into a 30 ga cannula $(18 \mathrm{~mm})$ and extended $2.0-2.5 \mathrm{~mm}$ beyond the distal end. The fine wire electrode cannula was affixed to a moveable microdrive that was constructed using a tapped carrier and threaded rods, which when turned lowered the electrodes through the brain. The impedance of the fine wire electrodes ranged from 100 to $400 \mathrm{k} \Omega$. The microdrive was also equipped with a 26 ga inf usion guide cannula $(20 \mathrm{~mm})$ that was positioned $0.5 \mathrm{~mm}$ lateral to and parallel with the fine wire electrode cannula.

\section{Surgery}

All rats received an oral antibiotic $(30 \mathrm{mg} / \mathrm{kg}$ Cefadroxil; Bristol-Myers, Barceloneta, PR) twice daily for $2 \mathrm{~d}$ before surgery. All surgeries were 
performed under aseptic conditions. The rats were anesthetized with sodium pentobarbital $(45 \mathrm{mg} / \mathrm{kg}$ ), and their body temperature was continuously monitored and maintained at $37 \pm 0.5^{\circ} \mathrm{C}$ using a rectal probe and thermal blanket (Harvard Apparatus, Edenbridge, KY). The rat's head was shaved and then positioned in a small animal stereotaxic apparatus (Kopf Instruments, Tujunga, CA). The scalp was cleansed with Betadine scrub and incised along the midline, and the skin and underlying fascia were gently retracted. Burr holes were drilled through the skull using a small drill mounted to the stereotaxic apparatus. The electrode microdrive was unilaterally implanted into mPFC in either the left $(n=4)$ or right $(n=5)$ hemisphere using the stereotaxic coordinates anteroposterior $+2.8 \mathrm{~mm}$, mediolateral $\pm 1.5 \mathrm{~mm}$, and dorsoventral -2.5 $\mathrm{mm}$ from the dura surface at a $10^{\circ}$ angle toward the midline, according to the rat brain atlas of Paxinos and Watson (1986). A Teflon-coated, stainless steel electrode $(250 \mu \mathrm{m}$; A-M Systems, Everitt, WA) was implanted into the contralateral sensorimotor cortex and served as a reference ground. A second Teflon-coated, stainless steel electrode $(250 \mu \mathrm{m})$ was attached to a machine screw on the skull surface and served as an animal ground. The microdrive and ground electrodes were connected to a plastic headstage connector, and all three were affixed to the skull surface with machine screws and dental acrylic. A stylet (32 ga) was placed into the infusion cannula to prevent obstruction. A topical gel containing a local anesthetic (lidocaine) and antibiotics (bacitracin, polymixin, and neomycin) was applied to the wound immediately after surgery and for $3 \mathrm{~d}$ postoperatively. All rats received the oral antibiotic twice daily for $3 \mathrm{~d}$ postoperatively.

\section{Postsurgical behavioral testing}

For 1 week after surgery, the rats were left in their home cage with free access to food and water. Thereafter, access to water was gradually reduced, and the rats were subsequently retrained to criterion performance on the sustained visual attention task. During postsurgical recovery and behavioral retraining, the microdrive was slowly advanced in 45 $\mu \mathrm{m}$ increments each day until the tips of the electrodes were positioned at the dorsal border of the prelimbic sector of the mPFC. Neurophysiological recording began after the rats had returned to the criterion level of performance. The rats were recorded and behaviorally tested 6-7 $\mathrm{d} /$ week and were given a minimum of three standard testing sessions between each distractor session.

\section{Neurophysiological recording}

Neural activity within the mPFC was recorded using pairs of electrodes configured as stereotrodes (McNaughton et al., 1983). The stereotrode signals were recorded using a four-channel operational amplifier that was fitted onto a headstage connector and cabled to a commutator that relayed the signals to a four-channel differential amplifier (A-M Systems). The analog signals were amplified $(10,000 \times)$, bandpass-filtered (low pass, $300 \mathrm{~Hz}$, high pass, $5 \mathrm{kHz}$ ), and then digitized by an analog-to-digital board (DT2821, $250 \mathrm{kHz}$ ). Only those signals exhibiting a peak amplitude, on either electrode of the stereotrode, that exceeded a user-defined threshold were sampled (at $25 \mathrm{kHz}$ ) and stored in $1.28 \mathrm{msec}$ windows using Discovery software (Datawave Technologies, Longmont, CO). Multiple unit activity on each stereotrode was separated into single units based on the clustering of signals after plotting the pair-wise relationships between various parameters extracted from the waveform on both electrodes of the stereotrode. The waveform parameters used for unit isolation included peak amplitude, peak phase angle, valley amplitude, valley phase angle, maximum peak to valley amplitude, and maximum spike width. Single unit separation was performed before the start of each behavioral testing session. Each set of isolated single units was recorded over the entire $46 \mathrm{~min}$ behavioral session and for a minimum of one distractor and three standard testing sessions. After characterization of a set of single units, the microdrive was incrementally advanced (45 $\mu \mathrm{m})$ and on the following day another set of single units was isolated from the multiple unit activity. Daily neurophysiological recording was continued over a 6-8 week period or until the tips of the stereotrodes were positioned at the midpoint of the prelimbic sector of the mPFC.

The timing of behavioral events was recorded simultaneously with the neurophysiological data. The operant software sent a $5 \mathrm{~V}$ pulse to a clock board at the onset of the signal light, tone, and houselight, at the time of right and left lever responses, at the time of water reinforcement delivery, and at the time of the water port entry. In addition, signals that coded the start and end of each trial, length of signal light illumination, and "nonsignal" events were also sent to the clock board. These behav- ioral events were inserted as flags into the neurophysiological data stream at a $0.1 \mathrm{msec}$ resolution for off-line correlation with single unit activity.

\section{Cholinergic deafferentation of $\mathrm{MPFC}$}

After neurophysiological characterization of mPFC single unit activity during attentional performance (which yielded an average of 33 units per rat across 26 standard and 9 distractor sessions), seven of the nine rats were given unilateral microinf usions of the cholinergic immunotoxin 192 IgG-saporin (Chemicon, Temecula, CA) into the recording area within the mPFC. The immunotoxin was diluted to a concentration of 0.05 $\mu \mathrm{g} / \mu \mathrm{l}$ in Dulbecco's saline. The rats were anesthetized with sodium pentobarbital $(45 \mathrm{mg} / \mathrm{kg})$, and a $32 \mathrm{ga}$ infusor was inserted into the $26 \mathrm{ga}$ infusion cannula affixed to the microdrive. The infusor tip extended 1 $\mathrm{mm}$ beyond the tip of the guide cannula and $\sim 1.3 \mathrm{~mm}$ above the tip of the recording electrodes. The infusor was connected via hypodermic polyvinylchloride tubing to a $10 \mu \mathrm{l}$ syringe that was mounted on an infusion pump (Harvard Apparatus, South Natwick, MA). A total volume of $1 \mu \mathrm{l}$ of the $192 \mathrm{IgG}$-saporin solution was injected at a rate of $0.2 \mu \mathrm{l} / \mathrm{min}$. After completion of the infusion, the infusor was left in place for an additional $5 \mathrm{~min}$. The two remaining rats served as unlesioned controls to allow for the direct assessment of the neurophysiological characteristics of ventrally situated $\mathrm{mPFC}$ units in nondeafferented rats to exclude the possibility that any alterations in mPFC electrophysiology evidenced during postlesion testing were related to an undocumented dorsal-ventral gradient in the characteristics of mPFC unit activity.

\section{Postlesion behavioral testing}

The rats were given a $3 \mathrm{~d}$ recovery period in their home cage with free access to food and water. Water restriction was then gradually implemented before the resumption of behavioral testing and neurophysiological recording, which continued for an additional 6-7 weeks.

\section{Electrolytic lesion}

At the conclusion of behavioral testing and neurophysiological recording, the rats were anesthetized with sodium pentobarbital $(45 \mathrm{mg} / \mathrm{kg})$, and the final recording site was marked with a small electrolytic lesion $(-15 \mu \mathrm{A}$ for $15 \mathrm{sec}$ ) using a stimulator and isolation unit (Grass Instruments, Quincy, MA). After the completion of the electrolytic lesion, the rats were prepared for transcardial perfusion.

\section{Histology}

The rats were perfused transcardially with $0.9 \%$ saline and then with $4 \%$ buffered paraformaldehyde solution. The brains were post-fixed for $24 \mathrm{hr}$ in $4 \%$ buffered paraformaldehyde and then transferred to a $25 \%$ sucrose-phosphate buffered solution until the time of sectioning. Free-floating sections $(40 \mu \mathrm{m})$ were taken from each brain using a cryostat, placed in $0.1 \mathrm{~m}$ phosphate buffer, $\mathrm{pH} 7.4$, and stored at $4^{\circ} \mathrm{C}$. A subset of the sections was processed for AChE histochemistry to determine the extent of cholinergic deafferentation, and the remaining sections were processed for cresyl violet staining to verify the placement of the recording electrodes within the mPFC.

AChE staining was conducted according to a modified version of the protocol by Tago et al. (1986) that has been described in detail by McGaughy et al. (1996), with the exception of the addition of the selective butyrylcholinesterase inhibitor, tetraisopropyl pyrophosphoramide $(0.137 \%)$, to the primary incubation media.

The remaining brain sections were processed for cresyl violet staining according to standard Nissl-staining procedures. Both sets of sections were evaluated for the extent of cholinergic deafferentation and electrode placement within the mPFC using light microscopy.

\section{Behavioral measures}

The behavioral measures generated for statistical analysis included response accuracy (\% correct) on signal trials as defined by the relative number of hits $([$ hits/(hits + misses $)] \times 100)$ and on nonsignal trials as defined by the relative number of correct rejections ([correct rejections/ (correct rejections + false alarms)] $\times 100$ ), response latency (latency from tone onset to lever press for each type of response), total errors of omission [(omits/total trials $) \times 100]$, errors of omission on signal [(signal trial omits/total signal trials $) \times 100]$ and nonsignal [(nonsignal trial omits/total nonsignal trials $\times 100]$ trials, and response lever side bias $[($ hits + false alarms $) /($ total \# of responses $)]$. The behavioral measures were generated from distractor testing sessions and the standard testing session just before the distractor session during both prelesion and 
postlesion testing. Standard testing sessions with $>60 \%$ overall omissions ( $1.8 \%$ of total) or distractor testing sessions with $>70 \%$ omissions within trial block 2 (13\% of total) were excluded from the statistical analyses.

\section{Neurophysiological measures}

Neurophysiological measurements were generated from single units exhibiting $>200$ total spikes during the recording sessions in which the behavioral performance met the criteria for behavioral analysis. The mean firing rate (spikes per second) of each single unit was determined over the entire 46 min testing session and from within each 5 min nontask period and each block of trials.

Peri-event time histograms (PETHs) were calculated for each neuron to determine the relationship between $\mathrm{mPFC}$ unit activity and behavioral performance during sustained visual attention. Individual single unit activity was summed in $20 \mathrm{msec}$ bins within a $\pm 1.5 \mathrm{sec}$ time frame around individual behavioral events during each testing session. The behavioral events included light onset at each signal length as well as collectively for all signal lengths, tone onset, hits, misses, correct rejections, false alarms, water delivery, water port entries, premature responses, ITI responses on the left lever, ITI responses on the right lever, and separately for the offset and onset of the houselight during distractor testing sessions.

The modulatory effect of the visual distractor on the firing rate of mPFC single units during distractor testing sessions was calculated by comparing the firing rate exhibited in trial blocks 1 and 3 with the firing rate during the presence of the flashing houselight in trial block 2 . If a unit exhibited at least a $15 \%$ alteration in the block 2 firing rate relative to the block 1 firing rate and subsequently exhibited at least a $10 \%$ alteration of firing in the opposite direction during block 3 relative to block 2 , then that unit was classified as exhibiting distractor-induced increases or decreases in firing based on the direction of the alteration during the presence of the visual distractor. The distractor-induced effects on firing rates were verified as general increases in unit activity and not alterations related to the onset and offset of the visual distractor by PETH analysis to the flashing houselight during trial block 2 . If a unit exhibited at least a $15 \%$ alteration in block 2 firing relative to block 1 but failed to exhibit at least a $10 \%$ reversal during block 3 , then that unit was classified as exhibiting task-induced increases or decreases in firing rate based on the direction of the alteration during the presence of the visual distractor. The percentage of single units exhibiting distractor-induced alterations in firing rate, task-induced alterations in firing rate, and no alterations in firing rate were determined from the total number of single units recorded. The magnitude of the distractor-induced alterations in single unit firing rates from block 1 to block 2 was also determined.

\section{Statistical analysis}

Behavioral data. Repeated-measures ANOVAs were conducted on the behavioral data using group (prelesion and postlesion), testing session (standard and distractor), and trial blocks (blocks 1-3) as within-subjects factors for the dependent measures of nonsignal response accuracy, errors of omission, response latency on nonsignal trials, and response lever side bias. The repeated-measures ANOVAs on the behavioral data from signal trials also included signal length $(25,50$, and $500 \mathrm{msec})$ as an additional within-subjects factor when analyzing the dependent measures of response accuracy, errors of omission, and response latency. In addition, separate repeated-measures ANOVAs were conducted on responses latencies during standard and distractor testing sessions and during the prelesion and postlesion phases of testing using response type (hit, correct rejection, miss, false alarm) as a within-subjects factor. Trial type-dependent errors of omission were also analyzed using a repeated design with group, testing session, and trial type (signal and nonsignal trials) as within-subjects factors. Post hoc analysis of significant main effects was performed using the Newman-Keuls test, whereas planned means comparisons were conducted to further analyze any significant interaction terms.

Neurophysiological data. Based on the established time course for cholinergic cellular degradation using 192 IgG-saporin (Torres et al., 1994; Waite et al., 1994), the postlesion neurophysiological recording data corresponded to the period from 2-7 weeks after 192 IgG-saporin infusion. Because a different set of units was recorded during postlesion testing relative to prelesion testing, a repeated-measures ANOVA was conducted on the overall firing rate of mPFC units with group (prelesion, unlesioned, and postlesion) as a between-subjects factor and task (pretask period and trial block 1) as a within-subjects factor, whereas post hoc analysis of significant group effects was performed using a NewmanKeuls test. Only those PETHs exhibiting phasic alterations in unit activ- ity proximate to individual behavioral events were tested for significant behavioral correlates using independent paired $t$ test analyses between the time frame encompassing the probable alteration in firing rate proximate to the behavioral event to an equivalent time frame temporally unrelated to the behavioral event. The effect of cholinergic deafferentation of the mPFC on the percentage of single units exhibiting significant behavioral correlates was examined using $\chi^{2}$ analyses by group. In addition, a repeated-measures ANOVA with group (prelesion and postlesion) and correlate (response-related and reward-related) as within-subjects variables was used to compare the magnitude of the decrease between the two major classes of behaviorally correlated unit activity after cholinergic deafferentation of mPFC. $\chi^{2}$ analyses were also performed on the percentage of single units that exhibited distractorinduced alterations in firing rate, whereas a one-way ANOVA by group was used to examine the magnitude of the distractor-induced alterations between the units recorded during prelesion, unlesioned, and postlesion phases of testing.

\section{RESULTS}

\section{Histology}

Verification of the placement of the recording electrodes was conducted on cresyl violet-stained brain sections. The final placement of the electrodes was determined by localizing the electrolytic lesion and reconstructing the dorsoventral path of the electrodes through the mPFC. The coronal section presented in Figure $2 A$ illustrates that the placement of recording electrodes in all rats was within the deep layers of mPFC (III-VI), and electrode path reconstruction revealed that all unit recordings were from neurons within the prelimbic sector of the mPFC.

Histological evaluation of the removal of cholinergic afferents within the mPFC was conducted on AChE-stained brain sections. Comparisons of AChE-positive fiber staining within the cortical layers of $\mathrm{mPFC}$ were made between the lesioned recording hemisphere and the intact, contralateral hemisphere. Six of the seven rats infused with $192 \mathrm{IgG}$-saporin exhibited a $\sim 75 \%$ decrease in the density of AChE-positive fiber staining throughout the anterior cingulate, medial precentral, prelimbic, and infralimbic sectors of the mPFC, with the most substantial loss evident within superficial cortical layers I and II and within deep layer VI of all mPFC regions (Fig. $2 B, C$ ). The remaining lesioned rat was excluded from the postlesion group because of decreases in the density of AChE-positive fiber staining within the recording hemisphere of $<75 \%$.

\section{Sustained attentional performance is unaffected by unilateral cholinergic deafferentation}

The detection of brief visual signals during attentional testing was signal length-dependent (signal length effect, $F_{(2,16)}=49.46, p<$ 0.001 ), with an increased relative number of hits occurring across increased lengths of signal presentations $(p<0.001)$ (Fig. 3). The presentation of a visual distractor (flashing houselight at $0.5 \mathrm{~Hz}$ during trial block 2) decreased the relative number of hits and correct rejections (testing session effect on hits and correct rejections, $F_{(1,8)}=30.35, p<0.001$, and $F_{(1,8)}=8.61, p<0.05$, respectively) (Fig. 4).

As predicted, unilateral cholinergic deafferentation of the mPFC was insufficient to affect attentional performance under standard or distractor testing conditions (Figs. 3, 4). The signal length-dependent detection of visual signals as well as the relative number of correct rejections did not differ between prelesion and postlesion testing under standard conditions. Likewise, distractorrelated decreases in hits and correct rejections were unaffected after the unilateral removal of cholinergic input to the mPFC. All other behavioral measures (errors of omission, response latency, 

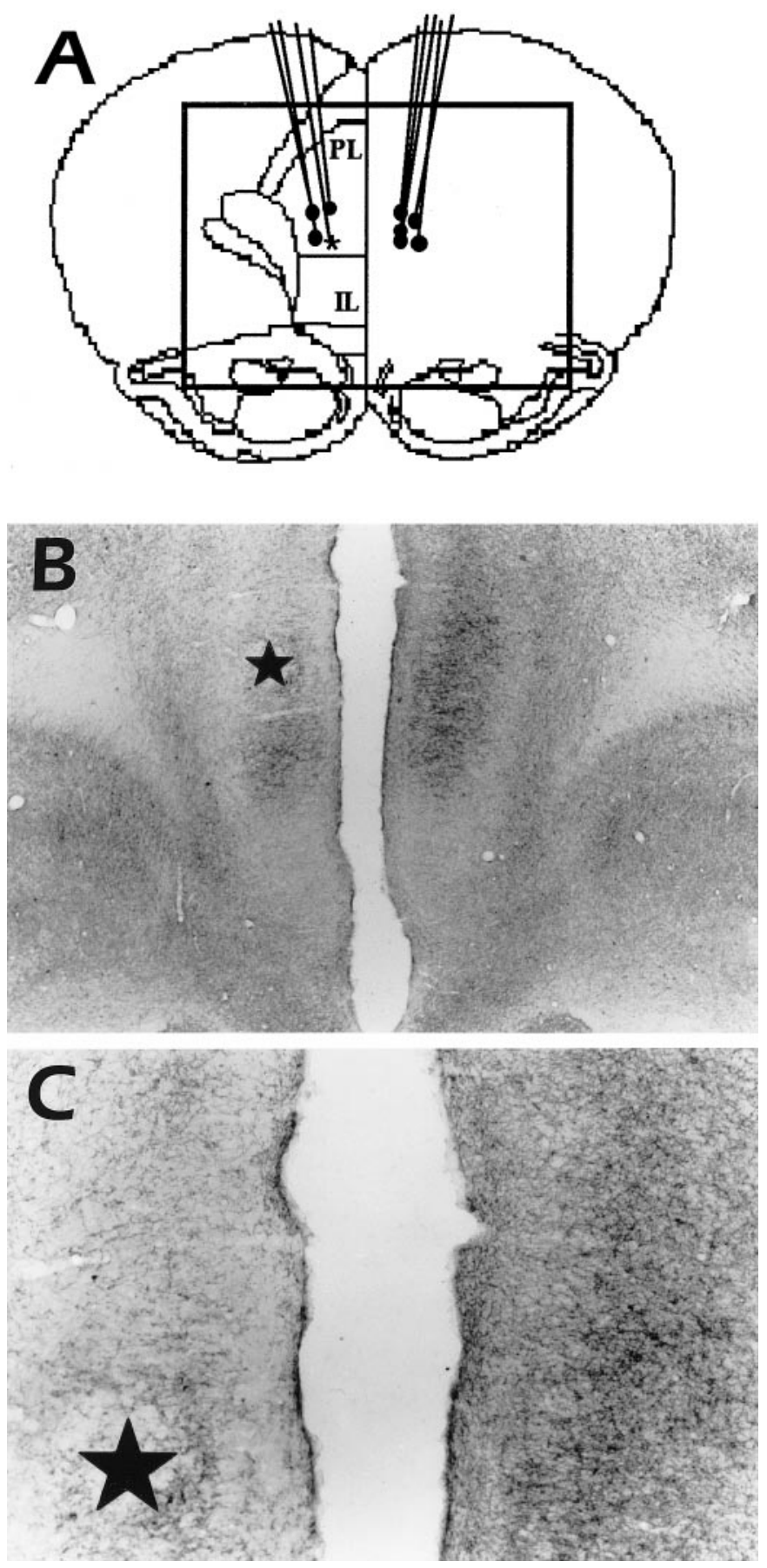

Figure 2. Electrode path and $192 \mathrm{IgG}$-saporin induced AChE-positive fiber loss within the mPFC. $A$, Schematic of a coronal section through the level of the mPFC (3.20 mm anterior to bregma) illustrating the path and final recording sites of the recording electrodes in the left or right hemispheres. $P L$, Prelimbic sector of mPFC; $I L$, infralimbic sector of mPFC. The box outline in $A$ represents the location from which the photomicrograph in $B$ was taken. $B$, Photomicrograph $(5 \times)$ illustrating the restricted loss of AChE-positive fibers to $\mathrm{MPFC}$ and the final recording site demarcated by the small electrolytic lesion (star) within mPFC layers III-V of the left hemisphere. $C$, Higher-magnification photomicrograph $(13.2 \times)$ of the loss of AChE-positive fibers throughout the recording area in $B$. Note the distinct loss of AChE-positive fiber staining throughout all cortical layers dorsal to the final recording site (star) when compared with the high density of fiber staining throughout the contralateral hemisphere.

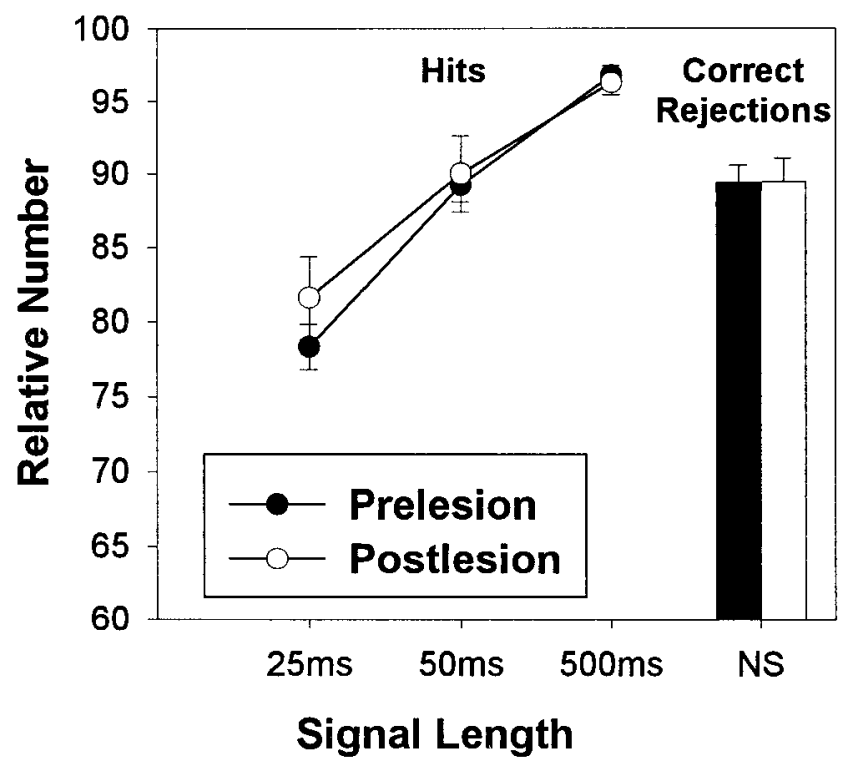

Figure 3. Sustained visual attention performance during baseline testing. Relative number of hits (mean \pm SEM) across signal lengths and relative number of correct rejections (mean \pm SEM) during prelesion (filled circles and bar) and postlesion (open circles and bar) phases of testing. NS, Nonsignal trials.

and response side bias) were similarly unaffected after unilateral cholinergic deafferentation.

\section{Distractor-induced modulation of mPFC unit firing}

During each recording session, three to eight units were isolated from the multiple unit activity recorded from each stereotrode, with a total of 257 units recorded during distractor testing sessions. The mean spontaneous firing rate $( \pm$ SEM) of mPFC units during the pretask period was $1.60 \pm 0.11$ spikes/sec, with a range from 0.04 to 12.67 spikes/sec. The firing rate of mPFC units was not significantly altered after the onset of behavioral performance within trial block $1(1.65 \pm 0.11$ spikes/sec, with a range from 0.03 to 17.70 spikes/sec).

The presence of the flashing houselight during distractor testing sessions modulated the firing rate of $18 \%$ of the mPFC units recorded during prelesion testing (Table 1). Twice as many units exhibited increased rates of firing compared with the number of units that exhibited decreased rates of firing during the presence of the visual distractor within the second block of trials relative to the rate of firing within trial block 1 . The firing rate of units positively modulated by the visual distractor had a mean percentage increase $( \pm$ SEM) of $81 \pm 14.0 \%$ during trial block 2 that reversed by an average of $24 \pm 2.2 \%$ after the offset of the visual distractor and return to standard testing conditions within the third block of trials. The mPFC units negatively modulated by the visual distractor exhibited a mean percentage decrease in firing $( \pm$ SEM) of $28 \pm 3.0 \%$ that reversed by an average of $33 \pm 12.4 \%$ after the offset of the visual distractor. The two units illustrated in Figure 5 exemplify the characteristic distractor-induced increases $(A)$ and decreases $(B)$ in unit firing.

In addition to distractor-induced modulations in the firing rate of mPFC units, a significant number of units $(30 \%)$ exhibited alterations in firing rate over the course of task performance that could not be solely attributed to the presence of the visual distractor (Table 1). By definition, these task-associated alterations in unit firing occurred during the second block of trials 

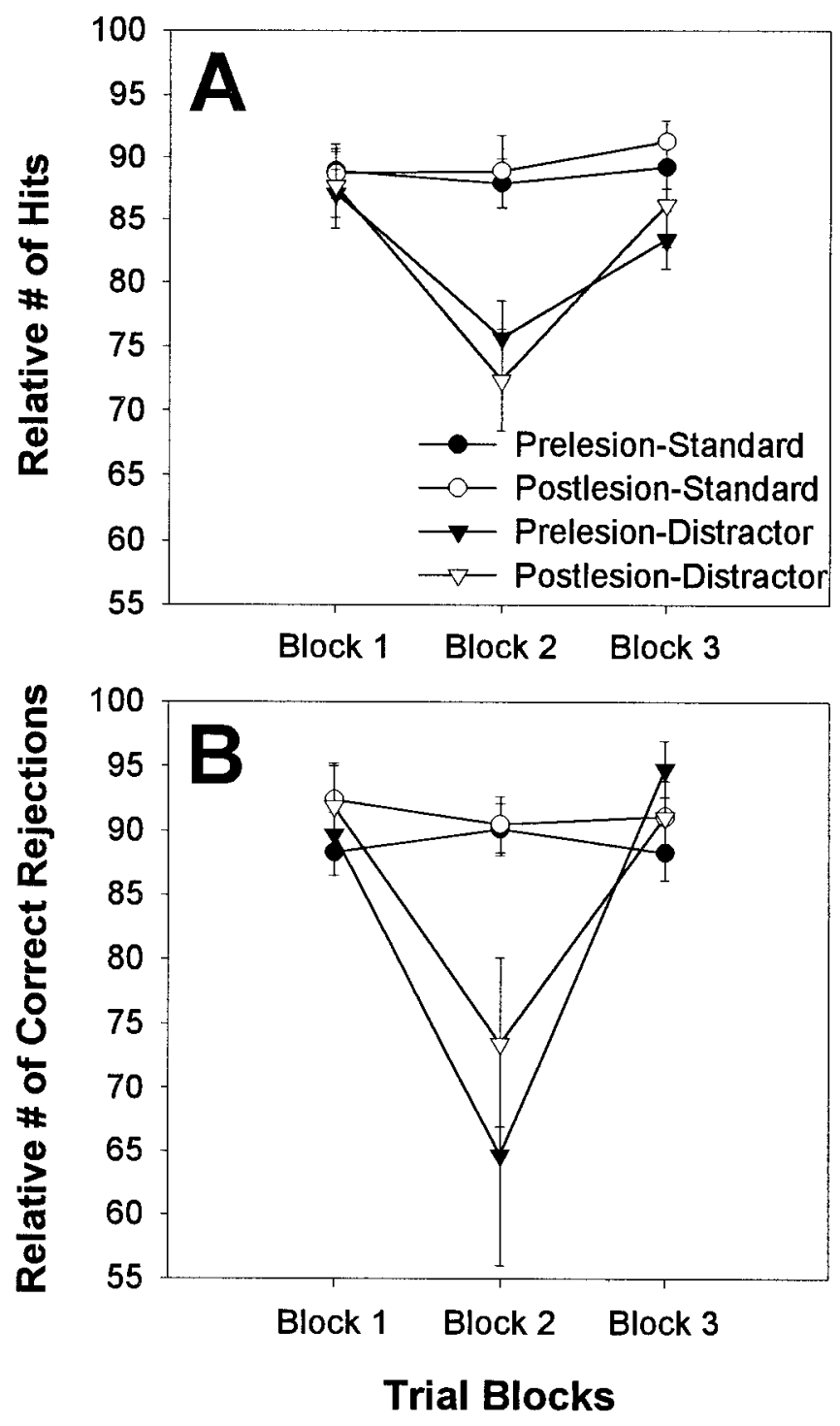

Figure 4. Sustained visual attention performance across trial blocks during standard and distractor testing conditions. $A$, Relative number of hits (mean \pm SEM) across trial blocks under standard and distractor testing conditions during prelesion and postlesion phases of attentional testing. $B$, Relative number of correct rejections (mean \pm SEM) across trial blocks under standard and distractor testing conditions during prelesion and postlesion phases of attentional testing.

( $\geq 15 \%$ increase or decrease), relative to the firing rate within trial block 1 , and either persisted or failed to reverse in direction ( $<10 \%$ change) within the third block of trials. In contrast to distractor-induced modulations in unit firing, approximately twice as many mPFC units exhibited task-associated decreases in firing rate relative to the number of units that exhibited taskassociated increases in the rate of firing. The firing rates of the remaining 136 units (53\% of the total) recorded during prelesion testing were not directly influenced by the presence of the visual distractor or task performance during distractor testing sessions.

Cholinergic deafferentation attenuates mPFC unit firing and distractor-induced increases in neuronal activity

An additional 197 units were recorded during the unlesioned (102 units) and postlesion (95 units) phases of attentional testing. The loss of cholinergic input within the recording field produced a
Table 1. Alterations in the firing rate of mPFC neural activity during distractor sessions of sustained visual attention

Testing phase

\begin{tabular}{lclc}
\cline { 2 - 4 } Alteration in firing rate & Prelesion & Unlesioned & Postlesion \\
\hline Distractor-induced increase & $12 \%(30)$ & $10 \%(10)$ & $3 \%(3)^{*}$ \\
Distractor-induced decrease & $6 \%(15)$ & $11 \%(11)$ & $12 \%(11)$ \\
Task-associated increase & $11 \%(28)$ & $14 \%(14)$ & $7 \%(7)$ \\
Task-associated decrease & $19 \%(48)$ & $21 \%(21)$ & $27 \%(26)$ \\
No alteration & $53 \%(136)$ & $44 \%(45)$ & $51 \%(48)$
\end{tabular}

Data are expressed as the percentage of units (absolute number noted in parentheses) displaying alterations in firing rate from the total number of units recorded (prelesion $=257$; unlesioned $=102$; postlesion $=95)$.

* Significantly different from prelesion $(p<0.05)$.

significant decrease $(52 \%)$ in the firing rate of mPFC units during task performance within trial block 1 (group effect, $F_{(2,451)}=$ 13.14, $p<0.001)$. Post hoc analysis revealed that the overall firing rate of $\mathrm{mPFC}$ units recorded after cholinergic deafferentation (0.79 spikes/sec) was significantly slower than the firing rate of units recorded during both the prelesion (1.65 spikes/sec) and unlesioned (1.45 spikes/sec) phases of testing $(p<0.05)$. In addition, the firing rate of units recorded during the prelesion and unlesioned phases of testing did not significantly differ, indicating that the decrease in unit activity during postlesion testing was caused by the loss of cholinergic input and not the result of an intrinsic difference in the firing rate of units situated more ventrally within the prelimbic sector of mPFC.

In addition to the decrease in the overall rate of firing, the removal of cholinergic input to $\mathrm{mPFC}$ produced a significant decrease in the number of units whose rate of firing was positively modulated by the visual distractor when compared with prelesion testing $(\chi=5.70, p<0.05)$ (Table 1$)$. The magnitude of distractor-induced increases in firing rate was also markedly attenuated after the removal of cholinergic input relative to prelesion testing (Fig. 6). In contrast, the proportion of units whose firing rates were negatively modulated by the presence of the visual distractor doubled during postlesion testing, but the magnitude of those distractor-induced decreases in unit firing was not altered by the removal of cholinergic input $(28.4 \pm 3.8 \%)$. The data collected from the unlesioned rats suggests, however, that there may be an increased proportion of units $(11 \%)$ negatively modulated by the visual distractor at more ventral levels of the mPFC.

The proportion of units exhibiting task-associated increases or decreases in firing rate during distractor testing sessions was not significantly affected by the removal of cholinergic terminals within mPFC. Likewise, cholinergic deafferentation did not alter the proportion of $\mathrm{mPFC}$ units in which the firing rate remained stable despite the presence of the visual distractor and throughout task performance.

\section{Behavioral correlates of mPFC unit activity}

Of the units tested, 213 (72\% of the total recorded during prelesion testing) exhibited significant phasic alterations in activity that correlated with specific behavioral events (Table 2). Alterations in unit firing were not observed in relation to stimulus presentation (signal lights or tone), but rather were correlated with the preparation and production of correct responding as well as the anticipation and consumption of subsequent water reward. Consequently, behaviorally correlated mPFC unit activity was classified into response-related and reward-related categories, 

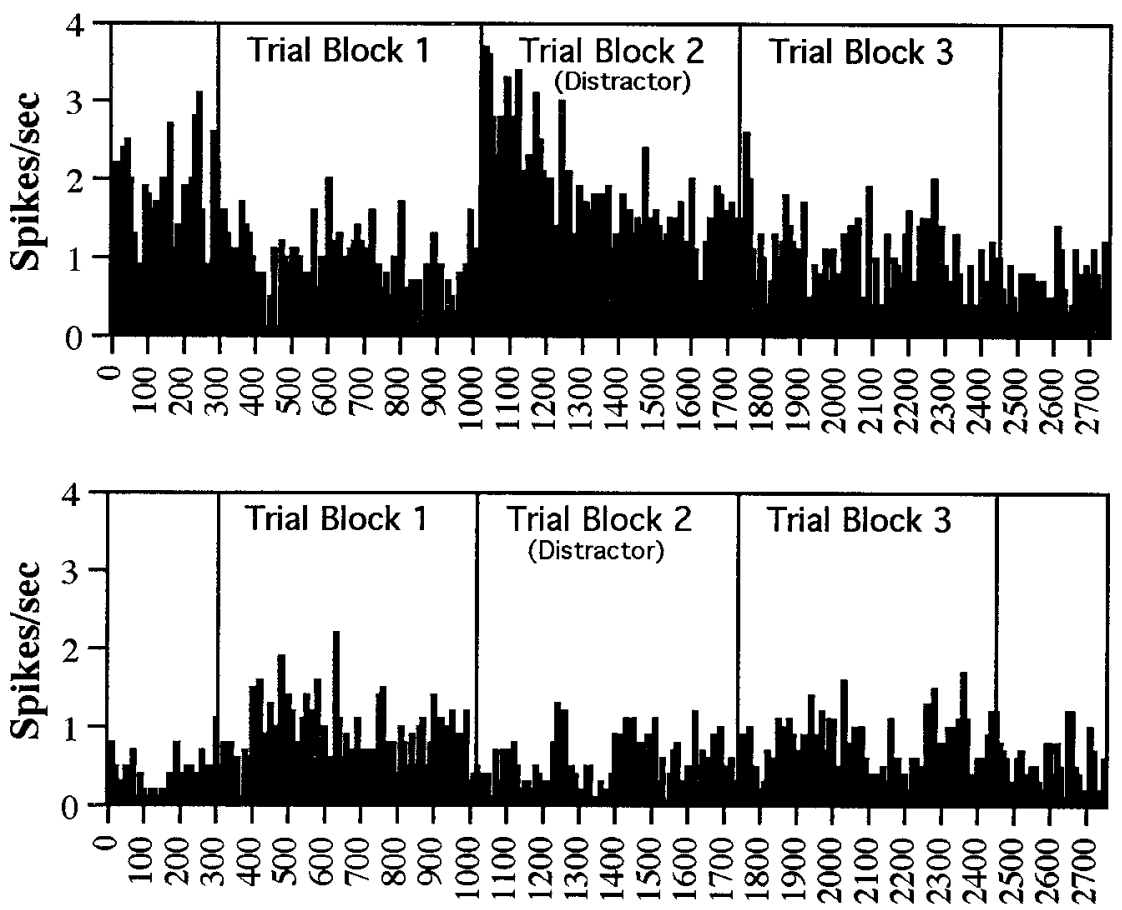

Time (sec)

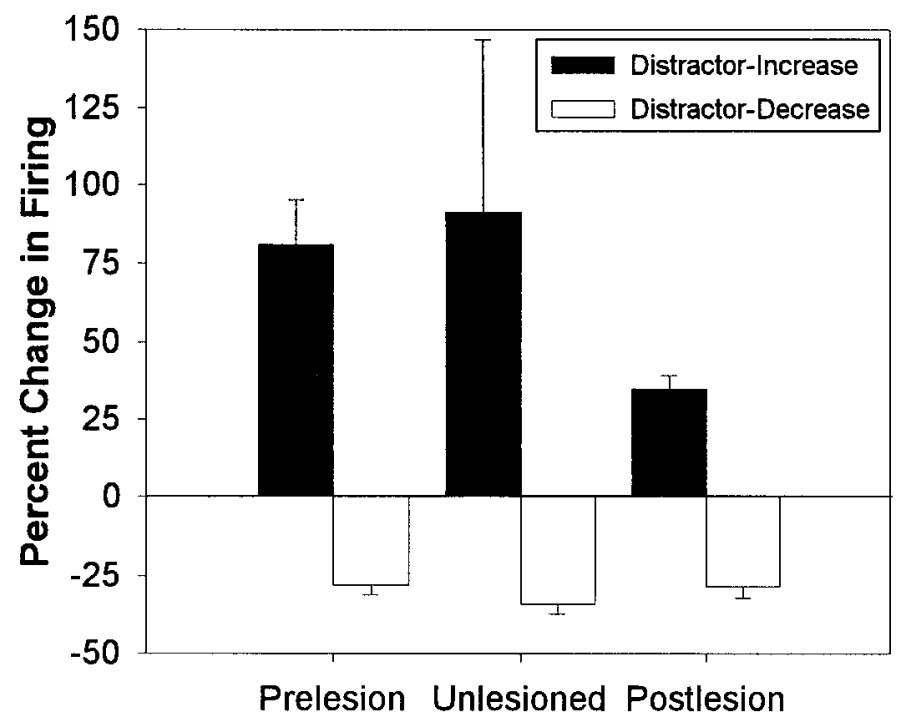

Figure 6. Amplitude of distractor-induced alterations in mPFC unit activity during sustained visual attention. The graph illustrates that the magnitude (mean \pm SEM) of positive alterations (percentage change, trial block 2 relative to trial block 1 ) in $\mathrm{mPFC}$ unit firing exhibited during the presence of the visual distractor was attenuated after the removal of cholinergic input, whereas the magnitude of negative alterations in unit firing during the presence of the visual distractor was unaltered across testing phases.

with approximately twice as many units exhibiting reward-related behavioral correlates compared with response-related behavioral correlates. In addition, the behavioral correlates were classified based on the direction of the phasic alteration in unit firing (excitatory or inhibitory). Figure 7 illustrates the PETHs of six different $\mathrm{mPFC}$ units recorded from the same rat but during different testing sessions that illustrate each class of behaviorally
Figure 5. Distractor modulation of mPFC unit activity. Mean firing rates of two mPFC units plotted over the course of a distractor testing session (46 min). $A$, Neuron that exhibited a distractor-induced increase in firing rate of $98 \%$ from 0.89 spikes/sec within trial block 1 to 1.76 spikes/sec during the presence of the visual distractor within trial block 2 that subsequently decreased by $55 \%$ to 0.97 spikes/sec after the return to standard testing conditions within trial block 3 . $B$, Neuron that exhibited a distractor-induced decrease in firing rate of $41 \%$ from 0.89 spikes/sec within trial block 1 to $0.52 \mathrm{spikes} / \mathrm{sec}$ during the presence of the visual distractor that subsequently increased by $38 \%$ to 0.72 spikes/sec within trial block 3 .
Table 2. Behavioral correlates of mPFC neural activity during sustained visual attention

\begin{tabular}{|c|c|c|c|}
\hline \multirow[b]{2}{*}{ Behavioral correlate } & \multicolumn{3}{|c|}{ Testing phase } \\
\hline & Prelesion & Unlesioned & Postlesion \\
\hline Response-related & $33 \%(99)$ & $41 \%(43)$ & $2 \%(3)^{*}$ \\
\hline Preresponse excitatory & $22 \%(64)$ & $24 \%(25)$ & $2 \%(2)^{*}$ \\
\hline Response excitatory & $8 \%(25)$ & $9 \%(9)$ & $1 \%(1)^{*}$ \\
\hline Response inhibitory & $13 \%(37)$ & $20 \%(21)$ & $0 \%(0)^{*}$ \\
\hline Trial-type specificity & $79 \%(78)$ & $72 \%(31)$ & $100 \%(3)$ \\
\hline Reward-related & $67 \%(197)$ & $67 \%(71)$ & $27 \%(34)$ \\
\hline \multicolumn{4}{|l|}{ Anticipatory } \\
\hline Excitatory & $33 \%(99)$ & $33 \%(35)$ & $9 \%(12) *$ \\
\hline Inhibitory & $8 \%(25)$ & $10 \%(11)$ & $1 \%(1)^{*}$ \\
\hline \multicolumn{4}{|l|}{ Consummatory } \\
\hline Excitatory & $45 \%(132)$ & $49 \%(52)$ & $17 \%(22)$ \\
\hline Inhibitory & $2 \%(6)$ & $5 \%(5)$ & $0 \%(0)$ \\
\hline Trial-type specificity & $35 \%(69)$ & $20 \%(14)$ & $24 \%(8)$ \\
\hline Multiple correlates & $54 \%(115)$ & $61 \%(46)$ & $3 \%(2)^{*}$ \\
\hline Total correlates & $72 \%(213)$ & $71 \%(75)$ & $28 \%(36)$ \\
\hline No correlates & $28 \%(83)$ & $29 \%(31)$ & $72 \%(91) *$ \\
\hline
\end{tabular}

Data are expressed as the percentage of units (absolute number noted in parentheses) displaying significant behavioral correlates from the total number of units recorded $($ prelesion $=296$; unlesioned $=106$; postlesion $=127$ ), except for the measures of trial-type specificity and multiple correlates, which are expressed as a percentage of units from the total number of units displaying that specific type of behavioral correlate and the total number of units displaying any correlate, respectively.

* Significantly different from both prelesion and unlesioned $(p<0.05)$.

correlated unit activity identified during prelesion attentional testing.

\section{Response-related firing of $\mathrm{MPFC}$ units}

One third of all the units recorded during prelesion testing exhibited response-related alterations in firing rate that were 

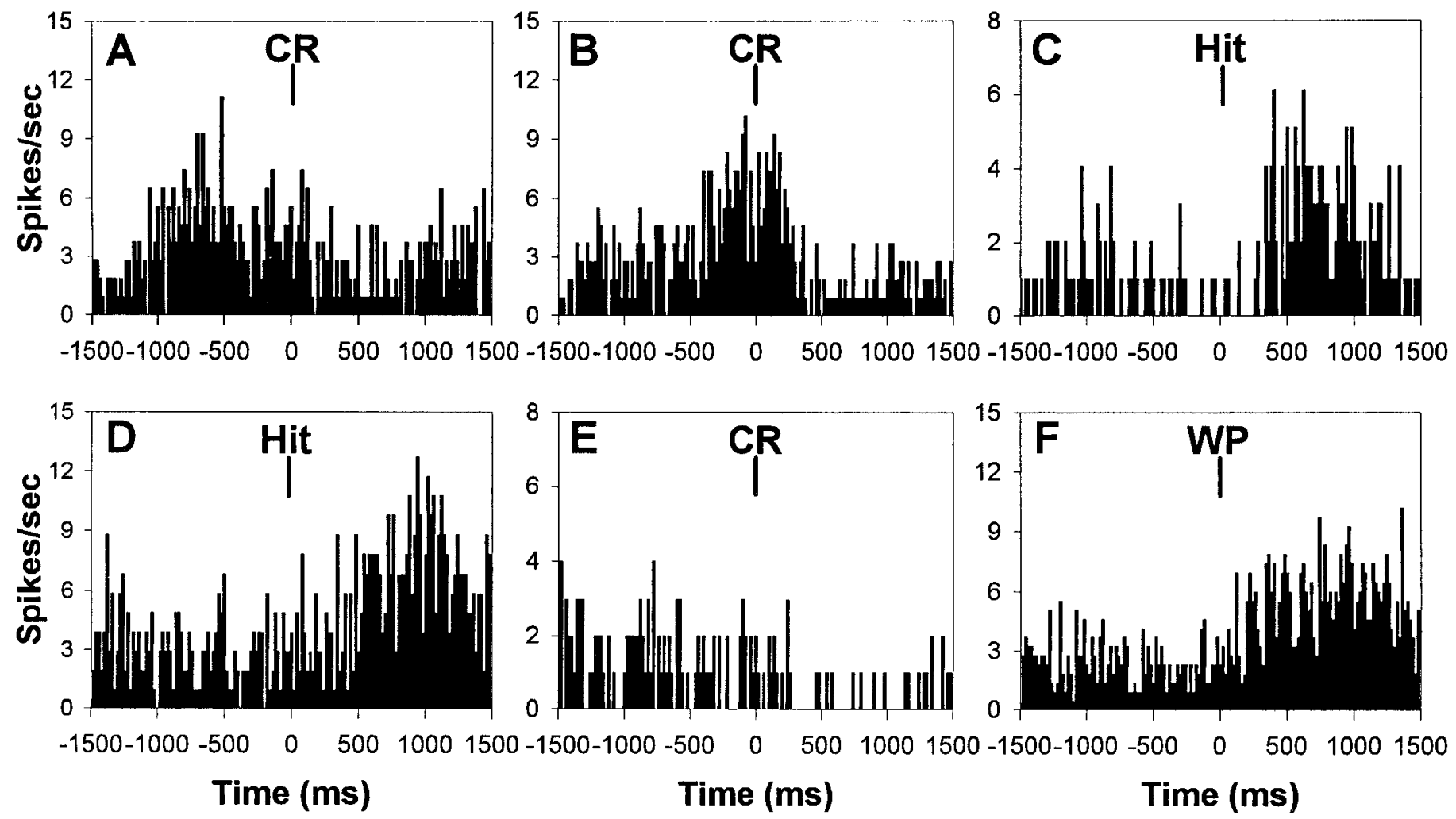

Figure 7. Behavioral correlates of mPFC unit activity during sustained visual attention. The PETHs (20 msec per bin) presented are from six different mPFC units at different levels within the prelimbic sector of the same rat. $A-C$, Representative response-related behavioral correlates. $A$ illustrates a preresponse excitatory correlate with increased unit firing before correct rejection responses ( $C R$, correct rejection); $B$ illustrates a response excitatory correlate with increased unit firing coincident with correct rejection responses; and $C$ illustrates a response inhibitory correlate with decreased unit firing coincident with hit responses. $D-F$, Representative reward-related behavioral correlates. $D$ illustrates an anticipatory excitatory correlate with increased unit firing that began shortly after the emission of hits and remained elevated until the rat reached the water port; $E$ illustrates an anticipatory inhibitory correlate with decreased unit firing after correct rejection responses and during the approach to the water port; and $F$ illustrates a consumption excitatory correlate with increased unit firing that occurred immediately after entry into the water port and remained elevated throughout the consumption of the reward. $W P$, Water port entry.

temporally correlated with either correct response preparation and/or correct response emission (Table 2). The most frequent response-related unit activity was an increased rate of firing just before the emission of a correct response, and this type of phasic activity was classified as a "preresponse excitatory" behavioral correlate (Fig. 7A). A phasic increase in the rate of firing concurrent with correct responding was also found in approximately one-fourth of all the units with response-related activity and was classified as a "response excitatory" behavioral correlate (Fig. $7 B$ ). In addition to increased rates of mPFC unit firing during correct responding, decreased unit activity was also exhibited simultaneous with the emission of correct responses, was slightly more frequent than instances of increased unit activity, and was classified as a "response inhibitory" behavioral correlate (Fig. 7C).

\section{Reward-related firing of $M P F C$ units}

Two-thirds of all the units recorded during prelesion testing exhibited significant phasic alterations in firing rate that were temporally correlated with either the anticipation or consumption of water reward or both. Half of all the units with reward-related activity exhibited an increased rate of firing after the emission of a correct response and during the approach to the water port, and that phasic increase in activity was classified as a "reward anticipation excitatory" behavioral correlate (Fig. 7D). A much smaller proportion of the reward-related units exhibited a decreased rate of unit firing after the emission of a correct response and during the approach to the water port that was classified as a "reward anticipation inhibitory" behavioral correlate (Fig. 7E).

The largest proportion of reward-related $\mathrm{mPFC}$ units exhibited phasic activity during the consumption of water reinforcement. The single most frequent type of phasic activity exhibited by $45 \%$ of all units recorded during prelesion testing was an increased rate of unit firing during the consumption of the water reward that was classified as a "reward consumption excitatory" behavioral correlate (Fig. $7 F$ ). In contrast to the large proportion of mPFC units that illustrated increased activity during reward consumption, very few units exhibited a decreased rate of firing during the consumption of the water reward.

\section{Trial-type specific activation of behaviorally correlated MPFC} unit activity

The vast majority of units identified with response-related phasic activity (79\%) demonstrated behaviorally correlated firing that was selectively exhibited during signal or nonsignal trial performance. Figure $8 A-C$ illustrates a response-related behavioral correlate that was selectively expressed during correct rejections on nonsignal trials but not during hits on signal trials or the same "correct rejection" lever press during the ITI. In the majority of single unit PETHs, the number of incorrect responses was too few to accurately assess correlated activity; however, in instances 

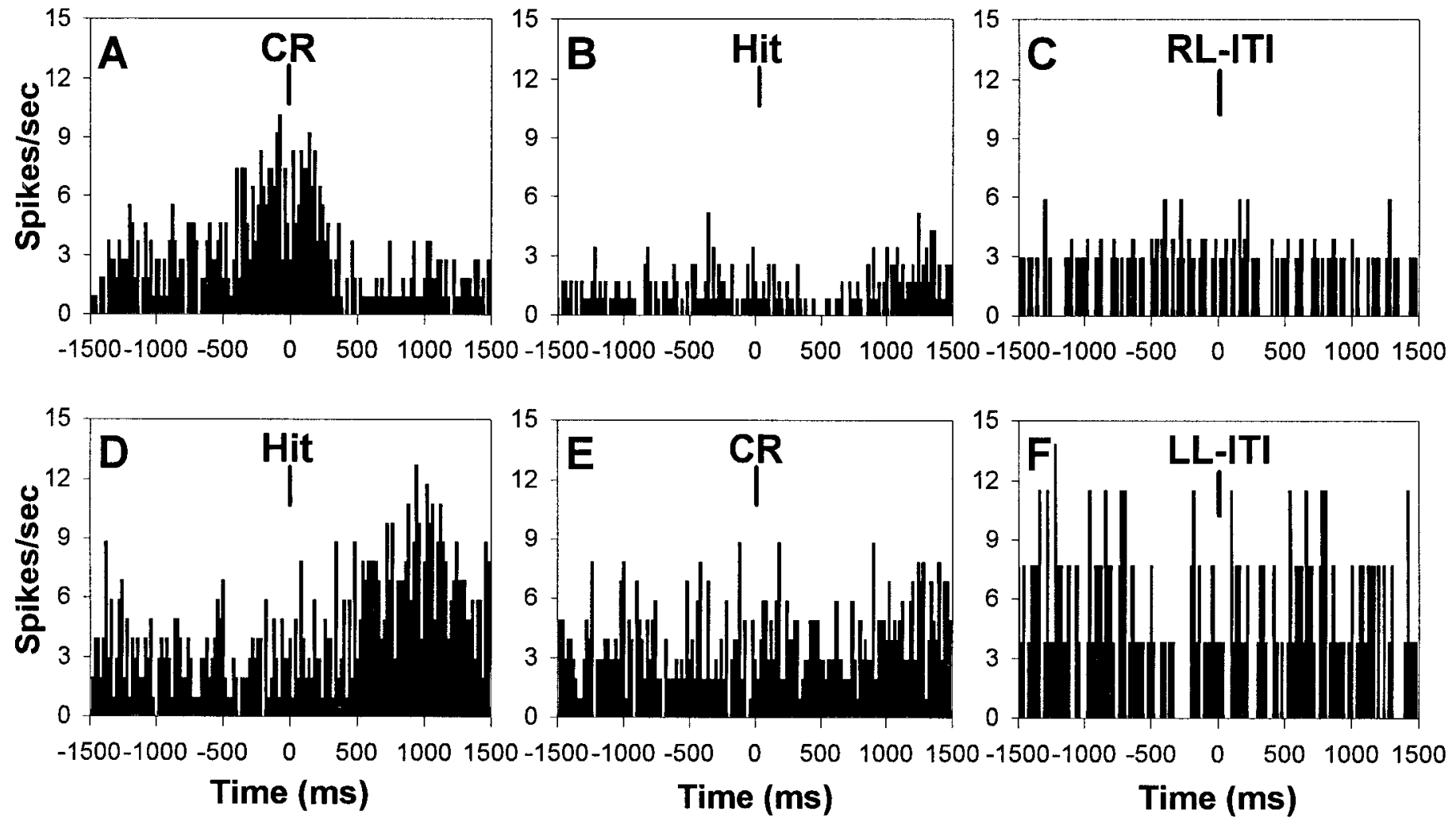

Figure 8. Trial-type specificity of behaviorally correlated mPFC unit activity during sustained visual attention. $A-C$, Trial type-specific unit activation of the same response-related behavioral correlate presented in Figure $7 B . A$ illustrates the PETH of the response excitatory correlate during correct rejections $(C R)$ on nonsignal trials, whereas $B$ illustrates the absence of behaviorally correlated activity from the same $\mathrm{mPFC}$ unit to hits on signal trials. Additionally, $C$ illustrates that responses on the same right lever as correct rejections, but during the intertrial interval $(R L-I T I)$, were not accompanied by increases in unit activity. $D-F$, Trial type-specific unit activation of the same reward-related behavioral correlate after signal trials presented in Figure 7D. $D$ illustrates the PETH of the reward anticipation excitatory correlate after hits on signal trials, whereas $E$ illustrates the absence of reward anticipatory activity after correct rejections on nonsignal trials. $F$ illustrates that responses on the same left lever as the hits, but during the intertrial interval $(L L-I T I)$, were not followed by increases in unit activity.

where response-related phasic activity was present and a substantial number of incorrect responses were emitted, the correlated activity was also observed during incorrect responding on the same response lever but not for responses on the same lever during the ITI.

Trial type-specific activation of reward-related behavioral correlates was less conspicuous than response-related correlates. Of the $197 \mathrm{mPFC}$ units identified as reward-related, roughly onethird demonstrated alterations in unit firing that were selective to correct responding on either signal or nonsignal trials. Figure $8 D-F$ illustrates a reward-related increase in unit activity during the approach to the water port after correct responses on signal trials that was not exhibited after correct responses on nonsignal trials or after lever presses on the same "hit" lever as signal trials but during the ITI. These results suggest that mPFC units can be differentially activated by afferent input that is driven by signal or nonsignal stimulus-response-reward associations.

\section{mPFC unit activity correlates with multiple behavioral events}

More than half of all the units recorded during prelesion testing displayed phasic activity that correlated with multiple behavioral events (Table 2). Figure $7 C$ illustrates an mPFC unit that had both a response inhibitory correlate with decreased unit activity during "hit" responding along with a reward anticipation excitatory correlate with increased unit activity during the approach to the water port. Multiple behavioral correlates were observed both within and across response-related and reward-related categories, suggesting that mPFC neural activity is inherently correlated with both the response production and reinforcement outcomes of sustained attentional performance.

\section{Behaviorally correlated MPFC unit activity is maintained during distractor-related behavioral impairments}

Behaviorally correlated mPFC unit activity exhibited under standard testing conditions is primarily unaltered by conditions of increased attentional demand despite the presence of distractorrelated decreases in the relative number of hits and correct rejections and modulations in the overall rate of unit firing (Fig. 9). This result suggests that behaviorally correlated mPFC unit activity is expressed independent from alterations in the overall rate of firing and may be responsible for the maintenance of behavioral performance under increased attentional demand, albeit at an impaired level relative to standard testing conditions.

\section{Cholinergic deafferentation markedly attenuates behaviorally correlated MPFC unit activity}

Cholinergic deafferentation of the mPFC produced a substantial decrease in the frequency of behavioral event-related neural activity during attentional performance. Table 2 illustrates that the proportion of mPFC units with behaviorally correlated alterations in unit firing significantly decreased from $72 \%$ during prelesion testing to $28 \%$ during postlesion testing $\left(\chi^{2}=7.15, p<\right.$ 

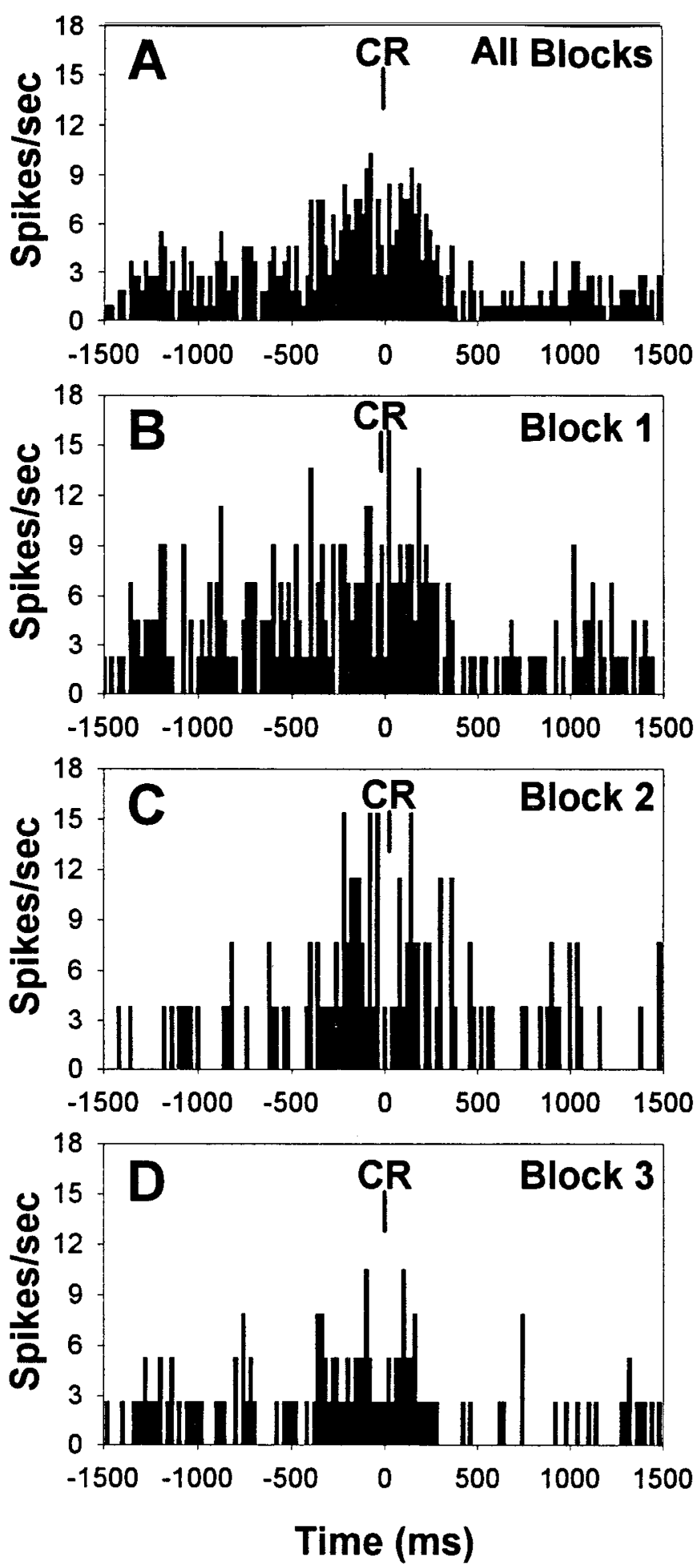

Figure 9. Behaviorally correlated $\mathrm{mPFC}$ unit activity during distractor testing sessions. $A$, PETH of the response excitatory correlate during correct rejections $(C R)$ summed across all three blocks of trials (same unit that was presented in Fig. $7 B$ ). $B-D$, PETHs of the same unit separated by trial blocks $1-3$. The increase in unit firing during correct rejection responses under standard testing conditions within trial blocks 1 and 3 ( $B$ and $D$, respectively) persisted despite the presence of the visual distractor within trial block $2(C)$ and concomitant decrease in the relative number of correct rejections and overall rate of firing (19\% decrease in overall firing rate in trial block 2 relative to block 1 ).
0.01). Analysis of the magnitude of the decrease between the two major classes of behavioral correlates revealed that the loss of cholinergic input did not differentially decrease the expression of response-related and reward-related unit activity $(p=0.90)$; however, in terms of absolute numbers, only three units exhibited response-related activity after cholinergic deafferentation, whereas one-fourth of the postlesion units exhibited reward-related activity that was principally expressed during reward consumption. In addition, the proportion of units exhibiting multiple types of behaviorally correlated unit activity was significantly attenuated by the loss of cholinergic input (Table 2). The loss of behaviorally correlated unit activity cannot simply be attributed to anatomical differences within the mPFC because data collected from units situated more ventrally within the prelimbic region of the unlesioned rats displayed the same distribution of behaviorally correlated activity, trial-type specificity, and multiple correlates as units recorded from more dorsal regions during prelesion testing.

\section{DISCUSSION}

The restricted removal of cholinergic fibers within the mPFC reduced the overall firing rate of units, attenuated the positive modulation of unit firing by the visual distractor, and diminished the phasic activity associated with specific behavioral events. The loss of the selective enhancement of neuronal activity during the visual distractor correlates with conditions of increased attentional demand and ACh efflux (Sarter et al., 1996) and further suggests that corticopetal cholinergic input from the BF is augmented under conditions of enhanced attention.

\section{Sustained attentional performance}

Sustained visual attentional performance was characterized by signal-length dependence of the hit rate and distractor-induced impairments on the relative number of both hits and correct rejections, all of which are consistent with previous findings using the same sustained attention task (McGaughy and Sarter, 1995; McGaughy et al., 1996, 1998). Attentional performance within this study was slightly more accurate than previous reports because of the slightly more stringent criterion levels implemented to elicit the high level of performance necessary for identifying behaviorally correlated unit firing.

As expected, unilateral cholinergic deafferentation of mPFC had no effect on attentional performance. The absence of a performance deficit after the unilateral lesion is consistent with the observation that widespread reductions in cortical $\mathrm{ACh}$, brought about by intracortical or BF infusions of 192 IgGsaporin, are required to produce impairments in this task (McGaughy et al., 1996; McGaughy and Sarter, 1998). Importantly, because the cholinergic lesion did not affect performance in the current study, alterations in the neurophysiological properties of mPFC neurons subsequent to cholinergic deafferentation cannot be attributed to altered behavioral performance but to the absence of ACh input.

\section{Attentional modulation of mPFC unit activity}

The enhanced attentional demand placed on the rat by the presence of the visual distractor was the most important behavioral factor influencing mPFC unit activity. The distractor also decreased the rate of responding and reduced the number of rewards. However, non-attentional variations in response rate and reward density, as well as the presentation of a "distractor" in a nonattentional context, do not alter cortical ACh release (Himmelheber et al., 1997). It is therefore unlikely that distractor- 
induced increases in mPFC unit activity were caused by changes in response rate or reward density, especially since mPFC unit activity has been examined under these same nonattentional conditions, and no such alterations in unit activity were observed (Hutchinson et al., 1996). Rather, attentional-related alterations in mPFC neural activity and the loss of that attentional modulation after the loss of cholinergic input correlate very strongly with the increase in ACh efflux that occurs within mPFC under conditions of enhanced attention (Sarter et al., 1996). Similar attentional demand-related increases in prefrontal unit firing have also been noted in monkeys performing an attentional task (Lecas, 1995). When the task was made more difficult by increasing the level of visual attention required for accurate performance, a population of neurons in prefrontal cortex exhibited increased levels of discharge that were proportional to the level of attention toward the stimulus.

\section{Neurophysiological properties of neurons within mPFC}

The activity of neurons within the deep layers of the prelimbic sector of mPFC were actively modulated by task variables during sustained attentional performance. The conspicuous lack of sensory-related activity is not surprising given the fact that unit recording began only after extensive training had occurred such that the stimulus-response relationships of the task were well entrained and the notion that prefrontal cortex is primarily involved with decisional and motor preparatory aspects of cognitive function, as well as the process of assigning behavioral significance to sensory stimuli (Diamond, 1990; Fuster, 1991; Balleine and Dickinson, 1998). Accordingly, mPFC neuronal activity was principally correlated with task variables relating to response and reward. As noted earlier, unit activity in some neurons was also behaviorally modulated by the presence of the visual distractor, correlating with changes in the attentional demands required by the task, and thus the level of attentional effort put forth by the rat.

The few studies that have investigated the behavioral and neurophysiological properties of mPFC (prelimbic) neurons in the rat have found similar associations of neural activity with movement and reward aspects of performance. During performance of spatial working memory tasks, most of the behaviorally correlated mPFC activity was goal-directed and movementrelated (Jung et al., 1998). Similarly, Poucet (1997) showed that mPFC neurons were not specifically affected by the rat's spatial location but rather were associated with food searching and exploratory behaviors. Neurons in the rat mPFC also become active before and during lever pressing for reward and in anticipation of reward delivery (Chang et al., 1997).

Neurons of the mPFC have been consistently reported to increase in activity before significant trial events. This activity has been hypothesized to underlie "anticipation" or "expectation" and has also been identified in primates. In recordings from the monkey dorsolateral prefrontal cortex, an area thought to be homologous to the rat mPFC (Uylings and van Eden, 1990; but see Preuss, 1995), neurons have been identified that not only are activated in anticipation of reward but are selective to specific types of reward (Watanabe, 1998). In the current results, rewardrelated correlates, both anticipatory and consummatory in nature, constituted the largest proportion of phasic unit activity, suggesting that the mPFC is part of the neural circuit that anticipates and monitors the outcomes of goal-directed behavior.

\section{Cholinergic modulation of mPFC unit activity}

Cholinergic deafferentation resulted in a general decrease in the overall responsiveness of $\mathrm{mPFC}$ neurons during task performance, an effect that is consistent with the identified actions of $\mathrm{ACh}$ in controlling the firing pattern and responsiveness of cortical neurons to sensory events and efferent input (Krnjevic et al., 1971; Spehlman et al., 1971; Stone, 1972; Bassant et al., 1990), although more complex interactions between excitatory and inhibitory functions of ACh do exist within cortical networks (McCormick and Prince, 1986; Xiang et al., 1998). The intracortical application of 192 IgG-saporin dennervated the entire mPFC, thereby preventing the determination of the effects of selective loss of ACh input on individual neurons from effects on the interaction between $\mathrm{mPFC}$ neurons; however, a preliminary analysis of ensemble coding and co-activity suggests that cholinergic deafferentation also produces substantial alterations in crosscorrelated activity patterns between neighboring $\mathrm{mPFC}$ neurons (Gill et al., 1998).

Although the effect of cholinergic deafferentation produced a loss in the attentional modulation of unit firing, this only applied to distractor-induced positive modulation of neuronal responses. The proportion of neurons that were negatively modulated by the distractor showed a strong trend toward increasing after the loss of cholinergic input. Increased cortical ACh efflux during the presence of a visual distractor (Sarter et al., 1996) suggests that the activity of BF corticopetal neurons may be augmented during times of enhanced attentional demand to facilitate the cortical processing of sensory information; however, when BF cortical cholinergic input to $\mathrm{mPFC}$ is selectively removed, as in the present study, modulation of cortical processing during increased levels of attention may still be influenced by BF corticopetal neurons that are GABAergic (Dykes, 1997; Gritti et al., 1997; Jimenez-Capdeville et al., 1997; Zaborsky et al., 1999). The presynaptic regulation of the BF corticopetal system may parallel that of the septohippocampal pathway (Smythe et al., 1992; Givens and Sarter, 1997), and as such, the cholinergic and GABAergic projections may work in concert to modulate cortical information processing.

The change in cholinergic afferent activity pursuant to increased attentional demand did not significantly influence the pattern of behaviorally correlated firing, suggesting that the specific input-output functions of $\mathrm{mPFC}$ neurons in processing information relevant to task events is not altered by enhanced ACh efflux, and thus attentional state. Perhaps this reflects the nature of the behavioral correlates observed, that is, response and reward-related. Most previous data concerning cholinergic modulation of unit activity has focused on the amplification of sensory signals (Dykes, 1997). After extensive cortical cholinergic deafferentation, the threshold for activation of mPFC neurons may become elevated such that the transfer of sensory information from posterior cortical regions can no longer trigger appropriate motor and reward-related unit activity, thereby accounting for the dramatic loss in the number of behavioral correlates observed.

\section{Conclusions}

The present data suggest that $\mathrm{mPFC}$ unit activity is related to sustained attentional performance and that corticopetal cholinergic projections may be critically involved in the generation of performance-related phasic activity. The correlation between enhanced cortical ACh release (Sarter et al., 1996) and the positive modulation of mPFC neuronal activity (present study) occurred against a background of impaired behavioral 
performance under high attentional demand; however, it may be that these same mechanisms maintain attentional performance at a maximum across varying levels of attentional demand. This hypothesis has recently been supported with evidence that bilateral cholinergic deafferentation of the $\mathrm{mPFC}$ results in impaired sustained visual attentional performance specifically under conditions of high attentional demand (Gill et al., 1999). Collectively, the present results along with other available evidence indicate a crucial role for the $\mathrm{BF}$ cholinergic system in the modulation of medial prefrontal cortical activity and mediation of attentional performance.

\section{REFERENCES}

Balleine BW, Dickinson A (1998) Goal-directed instrumental action: contingency and incentive learning and their cortical substrates. Neuropharmacology 37:407-419.

Bassant MH, Baleyte JM, Lamour Y (1990) Effects of acetylcholine on single cortical somatosensory neurons in the unanesthetized rat. Neuroscience 39:189-197.

Bucci DJ, Holland PC, Gallagher M (1998) Removal of cholinergic input to rat posterior parietal cortex disrupts incremental processing of conditioned stimuli. J Neurosci 18:8038-8046.

Chang JY, Zhang L, Janak PH, Woodward DJ (1997) Neuronal responses in prefrontal cortex and nucleus accumbens during heroin self-administration in freely moving rats. Brain Res 754:12-20.

Chiba AA, Bucci DJ, Holland PC, Gallagher M (1995) Basal forebrain cholinergic lesions disrupt increments but not decrements in conditioned stimulus processing. J Neurosci 15:7315-7322.

Chiba AA, Bushnell PJ, Oshiro WM, Gallagher M (1999) Selective removal of cholinergic neurons in basal forebrain alters cued target detection in rats. NeuroReport 10:3119-3123.

Diamond A (1990) The development and neural basis of memory functions as indexed by the $\mathrm{AB}$ and delayed response tasks in human infants and monkeys. In: The development and neural bases of higher cognitive functions (Diamond A, ed), pp 267-317. New York: New York Academy of Sciences.

Dykes RW (1997) Mechanisms controlling neuronal plasticity in somatosensory cortex. Can J Physiol Pharmacol 75:535-545.

Everitt BJ, Robbins TW (1997) Central cholinergic systems and cognition. Annu Rev Psychol 48:649-684.

Fuster JM (1990) Behavioral electrophysiology of the prefrontal cortex of the primate. Prog Brain Res 85:313-324.

Fuster JM (1991) The prefrontal cortex and its relation to behavior. Prog Brain Res 87:201-211.

Gill TM, Masters J, Sarter M, Givens B (1998) Correlated firing patterns of neighboring neurons within the rat medial prefrontal cortex during sustained visual attentional processing. Soc Neurosci Abstr 24:169.

Gill TM, Masters J, Sarter M, Givens B (1999) The role of acetylcholine within the medial prefrontal and posterior parietal cortices during sustained visual attention in the rat. Soc Neurosci Abstr 25:1895.

Givens B, Sarter M (1997) Modulation of cognitive processes by transsynaptic activation of the basal forebrain. Behav Brain Res 84:1-22.

Goldman-Rakic PS (1994) The issue of memory in the study of prefrontal function. In: Motor and cognitive function of the prefrontal cortex (Thierry AM, Glowinski J, Goldman-Rakic PS, Christen Y, eds), pp 112-123. Berlin: Springer.

Gritti I, Mainville L, Mancia M, Jones BE (1997) GABAergic and other noncholinergic basal forebrain neurons, together with cholinergic neurons, project to the mesocortex and isocortex in the rat. J Comp Neurol 383:163-177.

Himmelheber AM, Sarter M, Bruno JP (1997) Operant performance and cortical acetylcholine release: role of response rate, reward density, and non-contingent stimuli. Cognit Brain Res 6:23-36.

Himmelheber AM, Sarter M, Bruno JP (1998) Cortical acetylcholine release during performance of a vigilance task in rats. Soc Neurosci Abstr 24:2128.

Holley LA, Wiley RG, Lappi DA, Sarter M (1994) Cortical cholinergic deafferentation following the intracortical infusion of $192 \mathrm{IgG}$-saporin: a quantitative histochemical study. Brain Res 663:277-286.

Hutchinson K, Sarter M, Givens B (1996) Single unit activity in medial prefrontal cortex of rats performing an operant vigilance task. Soc Neurosci Abstr 22:1388.

Jimenez-Capdeville ME, Dykes RW, Myasnikov AA (1997) Differential control of cortical activity by the basal forebrain in rats: a role for both cholinergic and inhibitory influences. J Comp Neurol 381:53-67.

Jung MW, Qin Y, McNaughton BL, Barnes CA (1998) Firing characteristics of deep layer neurons in prefrontal cortex in rats performing spatial working memory tasks. Cereb Cortex 8:437-450.

Krnjevic K, Pumain R, Renaud L (1971) The mechanism of excitation by acetylcholine in the cerebral cortex. J Physiol (Lond) 215:247-268.

Lecas JC (1995) Prefrontal neurones sensitive to increased visual attention in the monkey. NeuroReport 7:305-309.

McCormick DA, Prince DA (1986) Mechanisms of action of acetylcholine in the guinea-pig cortex in vitro. J Physiol (Lond) 375:169-194.

McGaughy J, Sarter M (1995) Behavioral vigilance in rats: task validation and effects of age, amphetamine, and benzodiazepine receptor ligands. Psychopharmacology 117:340-357.

McGaughy J, Sarter M (1998) Sustained attention performance in rats with intracortical infusions of $192 \mathrm{IgG}$-saporin-induced cortical cholinergic deafferentation: effects of physostigmine and FG 7142. Behav Neurosci 112:1519-1525.

McGaughy J, Kaiser T, Sarter M (1996) Behavioral vigilance following inf usions of $192 \mathrm{IgG}$-saporin into the basal forebrain: selectivity of the behavioral impairment and relation to cortical AChE-positive fiber density. Behav Neurosci 110:247-265.

McNaughton BL, O'Keefe J, Barnes CA (1983) The stereotrode: a new technique for simultaneous isolation of several single units in the central nervous system from multiple unit records. J Neurosci Methods 8:391-397.

Muir JL, Dunnett SB, Robbins TW, Everitt BJ (1992) Attentional functions of the forebrain cholinergic systems: effects of intraventricular hemicholinium, physostigmine, basal forebrain lesions and intracortical grafts on a multiple-choice serial reaction time task. Exp Brain Res 89:611-622.

Muir JL, Page KJ, Sirinathsinghji DJS, Robbins TW, Everitt BJ (1993) Excitotoxic lesions of basal forebrain cholinergic neurons: effects on learning, memory and attention. Behav Brain Res 57:123-131.

Muir JL, Everitt BJ, Robbins TW (1994) AMPA-induced excitotoxic lesions of the basal forebrain: a significant role for the cortical cholinergic system in attentional function. J Neurosci 14:2313-2326.

Paxinos G, Watson C (1986) The rat brain in stereotaxic coordinates, Ed 2. Sydney: Academic.

Poucet B (1997) Searching for spatial unit firing in the prelimbic area of the rat medial prefrontal cortex. Behav Brain Res 84:151-159.

Preuss TM (1995) Do rats have prefrontal cortex? The Rose-WoosleyAkert program reconsidered. J Cognit Neurosci 7:1-24.

Public Health Service (1996) Public health service policy on humane care and use of laboratory animals. Washington, DC: U.S. Department of Health and Human Services.

Robbins TW (1996) Dissociating executive functions of the prefrontal cortex. Philos Trans R Soc Lond B Biol Sci 351:1463-1470.

Robbins TW (1997) Arousal systems and attentional processes. Biol Psychol 45:57-71.

Robbins TW, Everitt BJ, Marston HM, Wilkinson J, Jones GH, Page KJ (1989) Comparative effects of ibotenic acid- and quisqualic acidinduced lesions of the substantia innominata on attentional function in the rat: further implications for the role of the cholinergic neurons of the nucleus basalis in cognitive processes. Behav Brain Res 35:221-240.

Sarter M, Bruno JP (1997) Cognitive functions of cortical acetylcholine: towards a unifying hypothesis. Brain Res Rev 23:28-46.

Sarter M, Bruno JP (1999) Abnormal regulation of corticopetal cholinergic neurons and impaired information processing in neuropsychiatric disorders. Trends Neurosci 22:67-74.

Sarter M, Bruno JP, Givens B, Moore H, McGaughy J, McMahon K (1996) Neuronal mechanisms mediating drug-induced cognition enhancement: cognitive activity as a necessary intervening variable. Cogit Brain Res 3:329-343.

Sarter M, Bruno JP, Turchi J (1999) Basal forebrain afferent projections modulating cortical acetylcholine, attention, and implications for neuropsychiatric disorders. Ann NY Acad Sci 877:368-382.

Schoenbaum G, Eichenbaum H (1995) Information coding in the rodent prefrontal cortex. I. Single-neuron activity in orbitofrontal cortex compared with that in pyriform cortex. J Neurophysiol 74:733-750. 
Smythe JW, Colom LV, Bland BH (1992) The extrinsic modulation of hippocampal theta depends on the coactivation of cholinergic and GABA-ergic medial septal inputs. Neurosci Biobehav Rev 16:289-308.

Spehlman R, Daniels JC, Smathers CC Jr (1971) Acetylcholine and the synaptic transmission of specific impulses to the visual cortex. Brain 94:125-138.

Stone TW (1972) Cholinergic mechanisms in the rat somatosensory cerebral cortex. J Physiol (Lond) 225:485-499.

Tago H, Kimura H, Maeda T (1986) Visualization of detailed acetylcholinesterase fiber and neuron staining in rat brain by a sensitive histochemical procedure. J Histochem Cytochem 34:1431-1438.

Torres EM, Perry TA, Blockland A, Wilkinson LS, Wiley RG, Lappi DA, Dunnet SB (1994) Behavioural, histochemical and biochemical consequences of selective immunolesions in discrete regions of the basal forebrain cholinergic system. Neuroscience 63:95-122.

Turchi J, Sarter M (1997) Cortical acetylcholine and processing capacity: effects of cortical cholinergic deafferentation on crossmodal divided attention in rats. Cognit Brain Res 6:147-158.
Uylings HB, van Eden CG (1990) Qualitative and quantitative comparison of the prefrontal cortex in rat and in primates, including humans. Prog Brain Res 85:31-62.

Voytko ML (1996) Cognitive functions of the basal forebrain cholinergic system in monkeys: memory or attention. Behav Brain Res $75: 13-25$.

Waite JJ, Wardlow ML, Chen AC, Lappi DA, Wiley RG, Thal LJ (1994) Time course of cholinergic and monoaminergic changes in the rat brain after immunolesioning with $192 \mathrm{IgG}$-saporin. Neurosci Lett 169:154-158.

Watanabe M (1990) Frontal units of the monkey coding the associative significance of visual and auditory stimuli. Exp Brain Res 89:233-247.

Watanabe M (1998) Cognitive and motivational operations in primate prefrontal neurons. Rev Neurosci 9:225-241.

Xiang Z, Huguenard JR, Prince DA (1998) Cholinergic switching within neocortical inhibitory networks. Science 281:985-988.

Zaborsky L, Pang K, Somogyi J, Nadasdy Z, Kallo I (1999) The basal forebrain corticopetal system revisited. Ann NY Acad Sci 877:339-367. 